\title{
Speckle reduction in double-pass retinal images using variable-focus lenses
}

\author{
C. E. García-Guerra \\ carlos.enrique.garcia@cd6.upc.edu
}

\section{Aldaba}

M. Arjona

J. Pujol

\begin{abstract}
Centre of Sensors, Instruments and Systems Development (CD6). Polytechnic University of Catalonia (UPC). Rambla Sant Nebridi 10, 08222, Terrassa, Barcelona, Spain

Centre of Sensors, Instruments and Systems Development (CD6). Polytechnic University of Catalonia (UPC). Rambla Sant Nebridi 10, 08222, Terrassa, Barcelona, Spain

Centre of Sensors, Instruments and Systems Development (CD6). Polytechnic University of Catalonia (UPC). Rambla Sant Nebridi 10, 08222, Terrassa, Barcelona, Spain

Centre of Sensors, Instruments and Systems Development (CD6). Polytechnic University of Catalonia (UPC). Rambla Sant Nebridi 10, 08222, Terrassa, Barcelona, Spain
\end{abstract}

Speckle arises in double-pass images when coherent light is scattered by the retina. Since this noise degrades the images that are used to characterize the eye, there is special attention in reducing speckle when working with instruments based on retina reflections. In this work, we present a method for speckle reduction in double-pass retinal images by producing minor periodic variations in the vergence of the beam entering the eye with a variable-focus lens during image recording. Measurements in an artificial and a real eye following the implementation of the method corroborate the speckle reduction.

[DOI: http://dx.doi.org/10.2971/jeos.2015.15001]

Keywords: Speckle, visual optics, metrology

\section{INTRODUCTION}

The ability of the human visual system to process information from our surrounding is crucial to our daily activities. Unfortunately, the optical components of the eye are not perfect and present aberrations and intraocular scattering [1], which degrade the image of external objects formed on the retina. In this sense, the double-pass technique [2] is an excellent tool currently used in clinical environments to obtain an overall estimation of retinal optical quality [3]. This technique is based on projecting a point source on the retina and recording its aerial image after retinal reflection and double pass through the ocular media.

Double-pass retinal images are affected by speckle because they rely on reflections of coherent light in the retina, a rough surface composed of photoreceptors that scatter light back with a random phase [4]. The presence of speckle in doublepass images may alter the determination of parameters that describe the optical quality of the eye under assessment [2]. In consequence, speckle reduction is crucial to perform an accurate optical characterization when the eye is evaluated with instruments based on retinal reflection, such as the doublepass and Hartman-Shack [5] systems.

Speckle reduction is achieved if we add in an intensity basis a number of uncorrelated speckle patterns, which can be generated by time, space, or polarization diversity [6]. Since speckle structure in the eye presents a time-variant behaviour [2], the first methods to reduce this noise in double-pass images were based on time diversity by temporal integration in the recording or averaging a number of consecutive short-exposure frames [2]. Space diversity has also been explored in visual optics to reduce speckle by deviating rays reaching the retina with scanning mirrors [7], rotating diffusers [8], acoustic modulators [9] and broadband lasers [7].

In this research, we reduced speckle in double-pass retinal images by varying the vergence of the beam entering the eye with an electro-optical liquid lens, a device with a focal distance proportional to the applied current [10]. Similar to the use of broadband lasers where the speckle decorrelation is partially ruled by the defocus effect of the wavelength bandwidth [11], the induced vergence variations shift longitudinally the focal position of the beam in relation to the retina and change the optical path of reflected light [12]. This space diversity allows the generation of uncorrelated speckle patterns during the integration time of the recording device, permitting in this manner the speckle reduction. The effects of aberrations on speckle and the theory explaining this is widely documented [13]-[15]. From there, it is known that speckle is a function of defocusing for reflecting surfaces producing phase fluctuations $<\lambda / 2$, as in the case of the retina at $\lambda=780 \mathrm{~nm}$ [4]. Although the implementation of the method modifies the optical performance of the double-pass measurement system, the determination of the eye's optical quality is not compromised because the amplitude of the induced variations is such that the system can still be considered diffracted limited. Similar concepts have been applied to other areas of research [16]; however, to our best knowledge this method for speckle reduction has not yet been used in visual optics.

This article explains first the implementation of the method and the procedure that was followed to corroborate the 


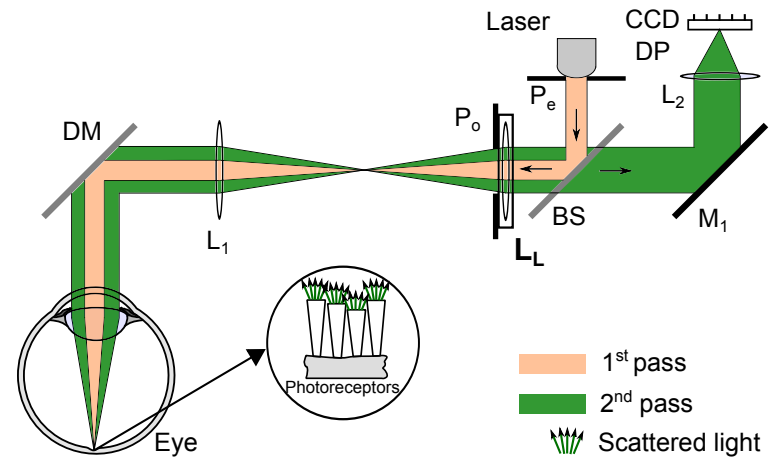

FIG. 1 Scheme of the double-pass system used during the experiments. Pupils: $P_{e}$, $P_{o}$; beam splitter: $B S$; focus-tunable lens: $L_{L}$; lenses: $L_{1}, L_{2}$; dichroic mirror: $D M$; mirror: $M_{1}$; camera: $C C D-D P$.

speckle reduction. It then presents the experimental results on an artificial and a real eye proceeded by a discussion on the applicability of the method in double-pass systems.

\section{EXPERIMENTAL PLATFORM}

The speckle reduction was measured with the experimental platform depicted in Figure 1. Light originating from a collimated laser diode (Monocrom MC7805U-M-7A15, $\lambda=780 \mathrm{~nm}$ ) reaches the pupil plane of the eye after passing through the $2 \mathrm{~mm}$ diameter circular diaphragm $P_{e}$ and the spherical refractive error corrector [17] composed by the commercially available variable-focus lens $L_{L}$ (Optotune EL-10-30-NIR-LD, $f_{L L}^{\prime}=36$ to $132 \mathrm{~mm}$ ) and the compensating lens $L_{1}$ which has a fixed focal distance of $f^{\prime}=50 \mathrm{~mm}$. After passing through the optics of the eye, the light is focused on the retina. There, some portion of this incident light is scattered by different structures of the retina and emitted towards the pupil [4], being this the beginning of the second pass. The portion of light that exists the eye is translated to the exit pupil plane by lens $L_{1}$. Here, the light reaches the $4 \mathrm{~mm}$ diameter pupil $P_{o}$ and then goes through the variable-focus lens and the beam splitter $B S$ before being focused by lens $L_{2}$ of $f^{\prime}=100 \mathrm{~mm}$ on the CCD camera (UEye UI-2220-M, pixel size of $8.3 \times 8.3 \mu \mathrm{m}$ ), which records the double-pass images.

We used the spherical refractive error corrector formed by the lens pair $L_{L}-L_{1}$ to generate the periodic vergence variations of small amplitude that are needed in the proposed method to produce the speckle reduction. The corrector is explained in detail in [17] and allows to set vergences to the beam entering the eye between +7.77 to -12.42 dioptres (D) by varying the current of the variable-focus lens $L_{L}$ with a sensitivity of $0.05 \mathrm{D} / \mathrm{mA}$. The variable lens is remote-controlled by a commercial driver (Optotune Electrical Lens Driver 4) capable of setting sinusoidal signals up to $v_{s}=100 \mathrm{KHz}$. This characteristic was used to configure powers in the beam entering the eye of the form $C=C_{0}+c \sin \left(2 \pi v_{s} t\right) \mathrm{D}$, where $C_{0}$ and $c$ are, respectively, the central optical power and the peak amplitude of the variations, $v_{s}$ is the frequency of the periodic signal, and $t$ represents time.

For comparison purposes during the quantification of the speckle reduction, the dichroic mirror $D M$ in front of the eye was mounted on a vibrating motor so that it is used as scanning mirror to break coherence and obtain non-speckled images. Real time video is available through a user interface developed in $\mathrm{C}++$. When image acquisition is requested, six consecutive images are recorded with an integration time of $40 \mathrm{~ms}$ and then post-processed in Matlab (MathWorks, 2010) following the procedure described in Section 4 of this document.

\section{EXPERIMENTAL PROCEDURE}

Two series of measurements were carried out under similar illumination conditions to corroborate the speckle reduction and the viability of the method. In the first series, we measured an artificial eye based on a lens of $f^{\prime}=50 \mathrm{~mm}$ with a cardboard at its focal distance acting as reflecting diffuser; a real eye of a healthy subject looking at infinity was analysed in the second series. Within each series, the central optical power $C_{0}$ of the spherical refractive error corrector was found by varying the current of the variable-focus lens until producing the image with the best optical quality (narrowest spot) with the scanning mirror switched on. Under these conditions, the power of the laser was configured to obtain non-saturated frames and then six consecutive images were recorded for each of the following combinations: scanning mirror on and $c=0$ (reference images $I_{0}$ ), scanning mirror off and $c=0$ (full speckle images $I_{S}$ ), and scanning mirror off and $c=0.25 \mathrm{D}$ (reduced speckle images $I_{R}$ ), where $c$ is the amplitude of the sinusoidal component of $C$ varying at $v_{s}=50 \mathrm{~Hz}$.

The amplitude of the variations was chosen based on simulations in Zemax (Zemax Development Corporation, 2013) showing that periodic changes in the vergence of the beam of $c=0.25 \mathrm{D}$ produce a Strehl ratio of 0.87 , which is a value higher than the Marechal criterion of 0.8 for diffractionlimited systems [18]. The frequency of variations is faster than the integration time of the camera and allows to record images that contain the effects of two cycles of the varying signal.

\section{DATA PROCESSING}

During data processing, $\bar{I}_{0}, \bar{I}_{S}$, and $\bar{I}_{R}$ were computed as the average of images $I_{0}, I_{S}$, and $I_{R}$, respectively. After that, the double-pass modulation transfer function MTF, the effective cut-off frequency $v_{c}$, and the Strehl ratio SR of the images and the averages were obtained following the flowchart provided in Figure 2. In this manner, the MTF was calculated as the radial profile of the Fourier transform of the images. Because applied to double-pass images captured with aperture diameters of 2 and $4 \mathrm{~mm}$ for the first and the second pass, the Fourier transform represents the product of the MTF for such pupil diameters $\left(M T F_{2} \times M T F_{4}\right)$ [19]. A peak correction [20] was performed by extrapolating the zero frequency from a curve fitting using a two-exponential function as proposed by Artal and Navarro [21]. After normalizing the curves, $v_{c}$ was computed by finding the frequency at which the MTF had a value of 0.01 . The SR was calculated as the ratio between areas under the MTF [22] of the image and that of the reference case $\bar{I}_{0}$, which we consider our benchmark. 


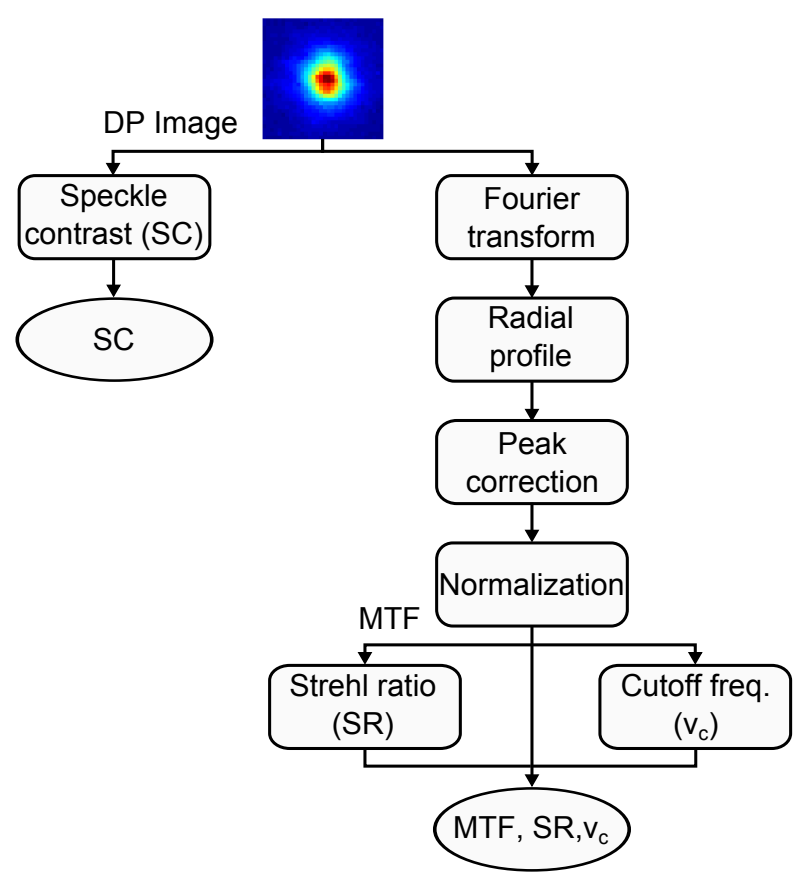

FIG. 2 Data processing flowchart followed to obtain the speckle contrast (SC), modulation transfer function (MTF), Strehl ratio (SR), and cut-off frequency $\left(v_{c}\right)$ from doublepass (DP) images.

The speckle was quantified in the images using the contrast of speckle $S C=\sigma / \bar{I}[6]$. This is the most commonly used metric to quantify this phenomenon and is defined as the ratio of the standard deviation $\sigma$ in the pattern to the average intensity $\bar{I}$. Based on the fact that double-pass images contain speckle in small areas, and that in diffracted limited cases with circular pupils they present circular symmetry, the SC was computed radially. First, the centre of mass of the image was estimated. Next, the mean and standard deviation of the intensities were obtained for all pixels whose round distance from the centre was equal to $d$ (in units of pixels). This process was done for integer distances $d$ from 0 to 16; in this manner, the computation covered the region where the effects of speckle were concentrated. We used the mean value of the contrast as metric to quantify speckle in the images and the relative error $e_{C}=\left(S C_{I}-S C_{\bar{I}_{0}}\right) / S C_{\bar{I}_{0}}$ to estimate deviations from $\bar{I}_{0}$.

The effects of the method in the spatial-frequency domain were determined by computing the errors in the parameters relative to those for $\bar{I}_{0}$, the average of the six images of the reference case $I_{0}$. Thus, we computed relative errors for the MTF $\left(e_{M}(v)\right)$, the mean value of $e_{M}(v)\left(e_{M}\right)$, the cut-off frequency $\left(e_{v}\right)$, and the Strehl ratio $\left(e_{S}\right)$ for $I_{0}, I_{S}$, and $I_{R}$ and the averages $\bar{I}_{0}, \bar{I}_{S}$, and $\bar{I}_{R}$. In all cases, the relatives errors were computed as $e_{\text {par }}=\left(\operatorname{par}_{I}-\operatorname{par}_{\bar{I}_{0}}\right) / \operatorname{par}_{\bar{I}_{0}}$, where par represents the parameters being calculated.

\section{RESULTS AND DISCUSSION}

The observed values of speckle contrast suggest that variations in the vergence of the beam during image recording led to speckle reduction. The mean SC for images $I_{S}, I_{R}$, and $I_{0}$ were $0.202(0.479), 0.171(0.378)$, and $0.124(0.295)$ for the artificial (real) eye, respectively. $I_{R}$ represents the double-pass images recorded following the implementation of the method

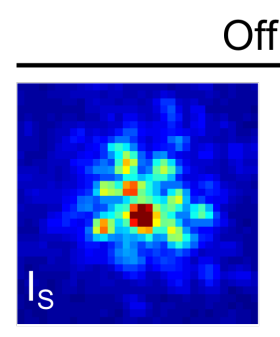

Off

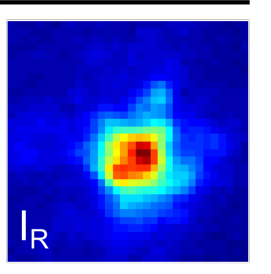

On
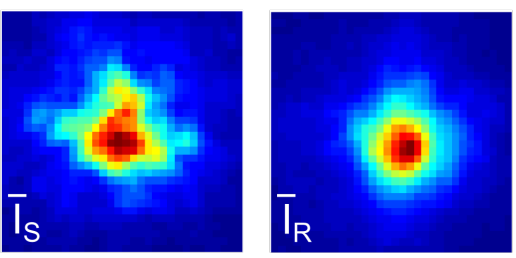

$I_{0}$

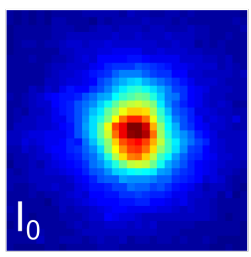

FIC. 3 First recorded $\left(I_{S}, I_{R}\right.$, and $I_{0}$; top) and average $\left(\bar{I}_{S}, \bar{I}_{R}, \bar{I}_{0}\right.$; bottom) doublepass images for the following cases: full speckle (sub-index $S$ ), reduced speckle (sub-index $R$ ), and reference (sub-index 0 ). On and $O f f$ refer to the states of the scanning mirror.

and we note that the mean speckle contrast of those recordings is lower than for $I_{S}$, which refers to images with full speckle. Furthermore, the magnitude of SC for $I_{R}$ is closer to that obtained for the reference images $I_{0}$. It is important to pay attention to the fact that the speckle contrast was computed radially. As a consequence, radial asymmetries in the images arising from eye aberrations may increase the magnitude of SC, without implying the presence of speckle. However, we consider that aberrations in the artificial eye did not play an important role. In the case of the real eye, visual inspection of images in Figure 3 with the scanning mirror switched on does not reveal asymmetries that could compromise the computation of SC. Although fluctuation of the accommodation in the real eye may also affect the quantification of speckle, the SC follows a similar behaviour than for the artificial eye, and we believe that differences between this parameter for images $I_{S}$, $I_{R}$, and $I_{0}$ are due to differences in the amount of contained speckle.

Figure 3 presents false coloured cropped $(32 \times 32)$ versions of the first recorded frame and the average of double-pass images $I_{S}, I_{R}$, and $I_{0}$ for the real eye. It is observed how the granularity in $I_{S}$ (full speckle) decreases in $I_{R}$ as consequence of applying our method for speckle reduction. A lower granularity is also observed in $\bar{I}_{S}$; since in the case of the real eye the speckle pattern changes with time, the averaging of short exposure images resulted in speckle reduction. The benefits of time diversity are also observed when comparing $I_{R}$ and the average $\bar{I}_{R}$. However, the spot shapes for images recorded during the application of the method are closer to the benchmark $\bar{I}_{0}$, even for the individual frame $I_{R}$. Double-pass images for the artificial eye are not presented here, but the speckle reduction was also recognized for images $I_{R}$. Since the artificial eye has a constant speckle pattern, there was not significant differences between the individual frames and the average images.

Figure 4 shows the double-pass MTF for image $\bar{I}_{0}\left(M T F_{\overline{0}}\right)$ and the mean relative error of the MTF for $I_{S}\left(e_{M S}(v)\right), I_{R}\left(e_{M R}(v)\right)$, and $I_{0}\left(e_{M 0}(v)\right)$ for the artificial and the real eye. The relative errors were computed as $e_{M I}(v)=\left(M T F_{I}-M T F_{\overline{0}}\right) / M T F_{\overline{0}}$, 

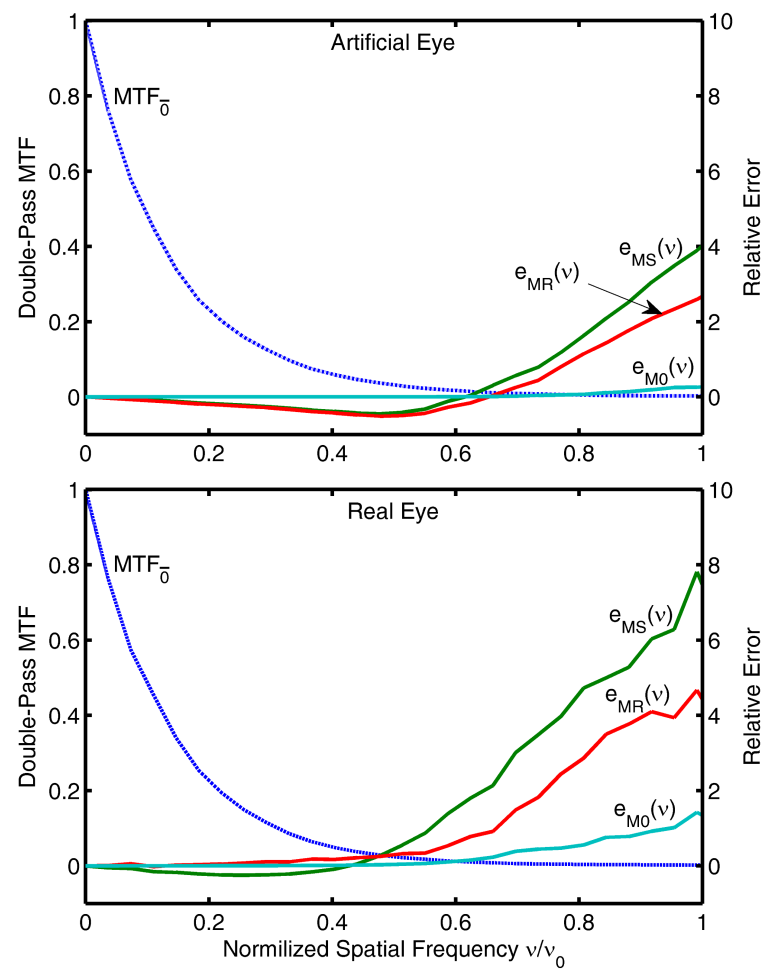

FIG. 4 MTF of the benchmark image $\bar{I}_{0}$ (discontinued line, left axis) and relative errors of the MTF for $I_{S}\left(e_{M S}(v)\right), I_{R}\left(e_{M R}(v)\right), I_{0}\left(e_{M 0}(v)\right)$ (continued curves, right axis) for the artificial (top) and real (bottom) eyes.

where sub-index $I$ represents the image under assessment. These errors represent a direct comparison of the MTF with respect to the benchmark $M T F_{\overline{0}}$ until the cut-off $v_{c}=P_{e} / \lambda=44.75 \mathrm{cyc} / \mathrm{deg}$ for the entrance pupil diameter $P_{e}=2 \mathrm{~mm}$. Referring to the curves in the figure, the negative values of $e_{M S}(v)$ indicate a decrement in the MTF at lower frequencies in images with full speckle. This behaviour is then reversed at higher frequencies, where the relative error goes above zero and the MTF presents the highest deviations. The differences between $e_{M S}(v)$ and $e_{M R}(v)$ reveal a reduction of the relative error for the measured eyes in images recorded following the implementation of the method. The performance of $e_{M 0}(v)$ indicates a small deviation of the MTF for images $I_{0}$ that is accentuated around the cut-off frequency, which may imply presence of speckle in the images despite the fact that they were recorded with the scanning mirror switched on. These tendencies are well reflected by $e_{M S}, e_{M R}$, and $e_{M 0}$. They represent the mean of the absolute value of the relative errors for images $I_{S}, I_{R}$, and $I_{0}$, respectively, and presented values of 1.199 (3.119), 0.837 (1.966), and 0.093 (0.475) for the artificial (real) eye.

Despite the magnitude that relative errors reach for images with full speckle in Figure 4 (peak values of more than 3 (7) for the artificial (real) eye), it should be highlighted that the MTF tends to zero at frequencies with higher affectations. However, it may also indicate that the presence of speckle can affect the determination of the effective cut-off frequency of the eye under assessment. Computed as the frequency at which the MTF had a value of 0.01 , it was found mean variations in terms of the cut-off $v_{c}$ ranging from 1.221 (1.345) to 0.869 (1.108) for the artificial (real) eye for images with full and reduced speckle. Furthermore, the lower values for images $I_{R}$ are closer to the cut-off frequency $0.686(0.633)$ obtained for the MTF of the benchmark image $\bar{I}_{0}$, which we consider without speckle. Observing the tendency of the values, the overestimation of the cut-off frequency is reduced when the method proposed in this work for speckle reduction is applied.

The computation of the Strehl ratio relative to that of image $\bar{I}_{0}$ did not reveal a clear tendency in the results. The mean relative Strehl ratio for images $I_{S}$ and $I_{R}$ went, respectively, from 0.966 (1.021) to 0.923 (1.107) for the artificial (real) eye; there is a decrement (increment) of the Strehl ratio and the values are lower (higher) than the ratio of the benchmark $\bar{I}_{0}$. This erratic behaviour can be inferred from the relative errors plotted in Figure 4. In the case of the artificial eye, errors $e_{M R}(v)$ and $e_{M S}(v)$ reflect that the MTF suffers a decrement at lower frequencies, which have the highest impact in the computation of the area, reducing in this manner the value of the Strehl ratio with respect the reference. On the other hand, the relative errors have such magnitude at the higher part of the spectrum for the real eye that the areas under the MTF experimented an overall increment with respect the area for the reference image $\bar{I}_{0}$, leading to relative Strehl ratios above one. We believe that the increment of the Strehl ratio for the real eye for images $I_{R}$ is related to a possible defocus during the recording of images $I_{0}$. According to Lohmann and Paris [23], it is possible to improve the MTF of a defocused lens by introducing certain amount of longitudinal vibrations. If it was the case, the vergence variations during the recording improved the optical quality of the eye, which is quantified as an increment of the Strehl ratio.

Table 1 synthesizes the mean relative errors for images $I_{S}, I_{R}$, and $I_{0}$, as well as the relative error for averages $\bar{I}_{S}, \bar{I}_{R}$, and $\bar{I}_{0}$. Since in the artificial eye the speckle pattern and aberrations are fixed, there are not significant differences in the errors computed from the images than from the averages. We consider that this fixed conditions during the measurements enable to relate the error variations to the effects of our method for speckle reduction. This situation may not be true in the real eye, where the time variant behaviour of the eye could influence the computation of the parameters. However, the metrics for the real eye presented similar performance than for the artificial one, which support the validity of the results. Under this assumption, the tendencies of the errors for the speckle contrast, MTF, and cut-off frequency indicate that the implementation of the method produced a speckle reduction.

The results discussed previously and summarized in Table 1 proof that speckle was reduced in the double-pass system by producing variations in the vergence during image recording. However, the speckle was not completely suppressed in the images under our measurement conditions. To increase the magnitude of the reduction we have to generate more uncorrelated speckle patterns by either increasing the amplitude of the vergence variations or by extending the image recording time. To deal with the decrements in image quality arising from the overall effects of the method, we could provoke a pre-defocus in order to compensate for the effects of the longitudinal vibrations induced by the vergence variations during the recording, as explained by [23]. Another option could be to compensate the decrements due to defocus during data 
processing by numerical focusing as done in digital holography [16]. However, a further research is needed to check the validity of these suggestions.

\begin{tabular}{|c|c|c|}
\hline & Artificial eye & Real eye \\
\hline \hline$e_{C S} / e_{C \bar{S}}$ & $0.801 / 0.759$ & $0.703 / 0.129$ \\
$e_{C R} / e_{C \bar{R}}$ & $0.528 / 0.489$ & $0.341 / 0.004$ \\
$e_{C 0} / e_{C \overline{0}}$ & $0.108 /-$ & $0.049 /-$ \\
\hline$e_{M S} / e_{M \bar{S}}$ & $1.199 / 1.172$ & $3.119 / 0.821$ \\
$e_{M R} / e_{M \bar{R}}$ & $0.837 / 0.836$ & $1.966 / 0.623$ \\
$e_{M 0} / e_{M \overline{0}}$ & $0.093 /-$ & $0.475 /-$ \\
\hline$e_{v S} / e_{v \bar{S}}$ & $0.780 / 0.766$ & $1.134 / 0.200$ \\
$e_{v R} / e_{v \bar{R}}$ & $0.267 / 0.221$ & $0.751 / 0.035$ \\
$e_{v 0} / e_{v \overline{0}}$ & $0.007 /-$ & $0.064 /-$ \\
\hline$e_{S S} / e_{S \bar{S}}$ & $0.035 / 0.040$ & $-0.021 / 0.066$ \\
$e_{S R} / e_{S \bar{R}}$ & $0.077 / 0.070$ & $-0.107 /-0.003$ \\
$e_{S 0} / e_{S \overline{0}}$ & $-0.012 /-$ & $-0.032 /-$ \\
\hline
\end{tabular}

TABLE 1 Relative errors of the speckle contrast $\left(e_{C}\right), \operatorname{MTF}\left(e_{M}\right)$, cut-off frequency $\left(e_{v}\right)$, and Strehl ratio $\left(e_{S}\right)$ for the full speckle (sub-indexes $S$ and $\bar{S}$ ), reduced speckle (sub-indexes $R$ and $\bar{R}$ ), and reference images (sub-indexes 0 and $\overline{0}$ ).

\section{CONCLUSION}

The results on both, the real and artificial eye confirmed that speckle can be reduced in double-pass images by inducing periodic defocuses of small magnitude with a variable-focusing lens. The variety of focal positions induced in this method during image recording is in some degree similar to that obtained for the different frequency components of broadband lasers, but produced with a lower cost. Compared to the use of scanning mirrors mounted on vibrating motors, the proposed technique does not produce mechanical vibrations that can be perceived by the subject and, with the time, misalign the optical components of the measuring system. The decreasing trends were observed for the artificial and the real eye. These results suggest that this method can be used in doublepass systems measuring the optical quality of the eye, since changes in focus did not compromise the optical characterization of the eye when they are lower than the $0.25 \mathrm{D}$ peak. We consider that the magnitude of the speckle reduction can be increased by producing pre-defocusing or by numerical focusing. Finally, we believe that this method to reduce speckle is also applicable to Hartmann-Shack systems.

\section{ACKNOWLEDGEMENTS}

This study was funded by the Spanish Ministry of Science and Innovation with the project grant DPI2011-30090-C02-01. The authors thank Ferran Sanàbria for his help with the experimental platform. Carlos E. García-Guerra acknowledges a Fellowship from the joint doctorate program EuroPhotonics. Final versions of this work were written at ITIV-KIT Karlsruhe Institute of Technology, Germany. Thanks to Wilhelm Stork for his kind support and to Nico Heußner for the critical reading of the manuscript.

\section{References}

[1] F. Díaz-Doutón, A. Benito, J. Pujol, M. Arjona, J. L. Güell, and P. Artal, "Comparison of the Retinal Image Quality with a HartmannShack Wavefront Sensor and a Double-Pass Instrument," Invest. Ophth. Vis. Sci. 47(4), 1710-1726 (2006).

[2] J. Santamaría, P. Artal, and L. Bescós, "Determination of the pointspread function of human eyes using a hybrid optical-digital method," J. Opt. Soc. Am. A 4, 1109-1114 (1987).

[3] J. L. Güell, J. Pujol, M. Arjona, F. Díaz-Doutón, and P. Artal, "Optical Quality Analysis System; Instrument for objective clinical evaluation of ocular optical quality," J. Cataract. Refr. Surg. 4, 1109-1114 (1987).

[4] S. Marcos, S. Burns, and J. C. He, "Model for cone directionality reflectometric measurements based on scattering," J. Opt. Soc. Am. A 8, 2012-2022 (1998).

[5] J. Liang, B. Grimm, S. Goelz, and J. F. Bille, "Objective measurement of wave aberrations of the human eye with the use of a HartmannShack wave-front sensor," J. Opt. Soc. Am. A 11, 1949-1957 (1994).

[6] J. W. Goodman, "Some fundamental properties of speckle," J. Opt. Soc. Am. 66, 1145-1150 (1976).

[7] H. Hofer, P. Artal, B. Singer, J. L. Aragón, and D. R. Williams, "Dynamics of the eye's wave aberration," J. Opt. Soc. Am. A 18, 497-506 (2001).

[8] K. M. Hampson, S. Chin, and E. A. H. Mallen, "Binocular ShackHartmann sensor for the human eye," J. Mod. Optic. 55, 703-716 (2007).

[9] V. Albanis, E. N. Ribak, and Y. Carmon, "Reduction of speckles in retinal reflection," Appl. Phys. Lett. 91, 054104 (2007).

[10] Optotune AG, Datasheet: EL-10-30-Series (Optotune AG, Dietikon, 2014).

[11] I. Yamaguchi, A. Yamamoto, and S. Kuwamura, "Speckle decorrelation in surface profilometry by wavelength scanning interferometry," Appl. Optics 32, 6721-6728 (1998).

[12] D. P. Kelly, J. E. Ward, U. Gopinathan, and J. T. Sheridan, "Controlling speckle using lenses and free space," Opt. Lett. 32, 3394-3396 (2007).

[13] H. M. Pedersen, "Theory of speckle dependence on surface roughness," J. Opt. Soc. Am. 66, 1204-1210 (1976).

[14] D. Kang, E. Clarkson, and T. D. Milster, "Effect of optical aberration on Gaussian laser speckle," Opt. Express 17, 3084-3100 (2009).

[15] H. M. Escamilla, E. R. Méndez, V. Ruiz-Cortés, and J. A. Landgrave, "Speckle contrast in the diffraction field of weak and strong diffusers in the presence of spherical aberrations," Opt. Commun. 313, 195-203 (2014).

[16] C. C. Yu, I. Matsubara, and W. J. Dallas, "Speckle noise reduction for digital holography using longitudinal shifting," in Biomedical Optics and 3-D Imaging: OSA Optics and Photonics Congress (OSA, Miami, 2012).

[17] F. Sanàbria, F. Díaz-Doutón, M. Aldaba, and J. Pujol, "Spherical refractive correction with an electro-optical liquid lens in a double-pass system," J. Eur. Opt. Soc.-Rapid 8, 13062 (2013).

[18] W. J. Smith, Modern Optical Engineering (McGrawHill, New York, 2000).

[19] P. Artal, S. Marcos, R. Navarro, and D. R. Williams, "Odd aberrations and double-pass measurements of retinal image quality," J. Opt. Soc. Am. A 12, 195-201, (1995). 
[20] A. Guirao, C. González, M. Redondo, E. Geraghty, S. Norrby, and P. Artal, "Average Optical Performance of the Human Eye as a Function of Age in a Normal Population," Invest. Ophth. Vis. Sci. 40, 203-213 (1999).

[21] P. Artal and R. Navarro, "Monochromatic modulation transfer function of the human eye for different pupil diameters: an analytical expression," J. Opt. Soc. Am. A 11, 246-249 (1994).
[22] M. Vilaseca, A. Padilla, J. C. Ondategui, M. Arjona, J. L. Güell, and J. Pujol, "Effect of laser in situ keratomileusis on vision analyzed using preoperative optical quality", J. Cataract. Refr. Surg. 36, 1945-1953 (2010).

[23] A. W. Lohmann and D. P. Paris, "Influence of longitudinal vibrations on image quality," Appl. Optics 4, 393-397 (1965). 